\title{
Platelet Dynamics in Peritoneal Carcinomatosis Patients Treated with Cytoreductive Surgery and Hyperthermic Intraperitoneal Oxaliplatin
}

\author{
Carlos Pérez-Ruixo, ${ }^{1}$ Belén Valenzuela, ${ }^{2,3,6}$ José Esteban Peris, ${ }^{1}$ Pedro Bretcha-Boix, ${ }^{2,3}$ \\ Vanesa Escudero-Ortiz, ${ }^{4}$ José Farré-Alegre, ${ }^{2,3}$ and Juan José Pérez-Ruixo ${ }^{5}$
}

Received 28 August 2015; accepted 15 October 2015; published online 17 November 2015

\begin{abstract}
The aim of the study was to characterize the platelet count (PLT) dynamics in peritoneal carcinomatosis patients treated with cytoreductive surgery (CRS) and hyperthermic intraperitoneal oxaliplatin (HIO). Data from patients treated with CRS alone $(N=18)$ or CRS and HIO $(N=62)$ were used to estimate the baseline platelet count $\left(\mathrm{PLT}_{0}\right)$, rate constants for platelet maturation $\left(k_{t r}\right)$ and platelet random destruction $\left(k_{s}\right)$, feedback on progenitor cell proliferation $(\gamma)$, and the drug-specific model parameters $(\alpha, \beta)$. Plasma oxaliplatin concentrations, $C_{p}$, reduced the proliferation rate of progenitor cells $\left(k_{\text {prol }}\right)$ according to a power function $\alpha \times C_{p}{ }^{\beta}$. The surgery effect on $k_{\text {prol }}$ and $k_{s}$ was explored. The typical values (between subject variability) of the $\mathrm{PLT}_{0}, k_{t r}, k_{s}, \gamma, \alpha$, and $\beta$ were estimated to be $237 \times 10^{9}$ cells/L $(32.9 \%), 7.09 \times 10^{-3} \mathrm{~h}^{-1}$ $(47.1 \%), 8.86 \times 10^{-3} \mathrm{~h}^{-1}(80.0 \%), 0.621,0.88 \mathrm{~L} / \mathrm{mg}(56.9 \%)$, and 2.63 . Surgery induced a maximal 2.09 -fold increase in $k_{\text {prol }}$ that was attenuated with a half-life of 8.42 days. Splenectomy decreased $k_{s}$ by $47.5 \%$. Age, sex, body surface area, sex, total proteins, and HIO carrier solution did not impact the model parameters. The model developed suggests that, following CRS and HIO, thrombocytopenia and thrombocytosis were reversible and short-lasting; the severity of the thrombocytopenia and thrombocytosis was inversely correlated, with splenectomized patients having thrombocytopenia of lower severity and thrombocytosis of higher severity; and the HIO dose and treatment duration determine the severity and duration of the thrombocytopenia. Higher HIO dose or longer treatment duration could be used without substantially increasing the risk of major hematological toxicity.
\end{abstract}

KEY WORDS: cytoreductive surgery; hyperthermic intraperitoneal chemotherapy (HIPEC); oxaliplatin; peritoneal carcinomatosis; population pharmacokinetic pharmacodynamic modeling; thrombocytopenia; thrombocytosis.

\section{INTRODUCTION}

Peritoneal carcinomatosis (PC) is an abdominal dissemination of malignant gastrointestinal and gynecological tumors, with or without evidence of metastatic systemic disease, which results in poor prognosis (1). The combination of cytoreductive surgery (CRS) and hyperthermic intraperitoneal oxaliplatin (HIO) is a promising strategy

\footnotetext{
${ }^{1}$ Pharmacy and Pharmaceutical Technology Department, University of Valencia, Valencia, Spain.

${ }^{2}$ Platform of Oncology, Hospital Quirón Torrevieja, Partida de la Loma s/n, 03184, TorreviejaAlicante, Spain.

${ }^{3}$ Cathedra of Multidisciplinary Oncology, UCAM Catholic University of San Antonio, Murcia, Spain.

${ }^{4}$ Pharmacy and Clinical Nutrition Group, University CEU Cardenal Herrera, ElcheAlicante, Spain.

${ }^{5}$ Clinical Pharmacology \& Pharmacometrics, Janssen Research \& Development, Beerse, Belgium.

${ }^{6}$ To whom correspondence should be addressed. (e-mail: belen.valenzuela@quiron.es)
}

for PC treatment (2) since retrospective and prospective analyses, including phase II and phase III clinical trials, as well as meta-analysis, have evidenced its survival benefit, relative to standard palliative surgery and/or chemotherapy (SPSC), in patients with resectable PC of colorectal origin $(3,4)$. The efficacy of this innovative treatment has also been reported in phase II studies in ovarian cancer $(5,6)$ and in phase III studies in gastric cancer $(7,8)$. These results encourage the clinical application of this aggressive treatment, particularly in conditions such as PC of nongynecologic origin, where the long-term survival is hardly ever seen after SPSC $(9,10)$.

Oxaliplatin is one of the most commonly used drugs in hyperthermic intraperitoneal chemotherapy because it has the ability to act at any stage of malignant cell replication (11), its intra-tumoral penetration is optimal (12), and its cytotoxicity is substantially improved by hyperthermia (13). Several phase I dose-escalation studies in PC patients treated with CRS have characterized the HIO pharmacokinetics in both the peritoneum and plasma (14-16). Following $460 \mathrm{mg} / \mathrm{m}^{2}$ dosing, the maximum HIO concentration $\left(C_{\max }\right)$ in the peritoneum $(330 \mathrm{mg} / \mathrm{L})$ (16) was 130 -fold 
higher than plasma $C_{\max }(2.59 \mathrm{mg} / \mathrm{L})$ after intravenous (IV) administration of $130 \mathrm{mg} / \mathrm{m}^{2}$ (17), indicating that, relative to IV dosing, HIO administration after CRS delivers a higher peritoneal oxaliplatin exposure with a minimum access to the systemic circulation, that limits the hematological toxicities, such as thrombocytopenia and neutropenia, which are the oxaliplatin dose-limiting toxicities after IV dosing (18). In a previous pharmacokinetic and pharmacodynamic (PK/PD) analysis, we characterized the immediate neutrophilia response induced by CRS and the HIO-induced myelosuppression (19). In the current manuscript, we aimed to describe the platelet dynamics in PC patients treated with CRS and HIO.

Platelets play a pivotal role in hemostasis after surgery (20). After HIO, platelet counts decline due to a myelosuppressive effect of the chemotherapy in the bone marrow, which may increase the bleeding risk, the platelet transfusions, the duration of hospitalization, and the postsurgical mortality $(9,10,12,21)$. Several days after a major surgery, a thrombocytosis, especially in patients with splenectomy, has been reported $(22,23)$ as part of the natural defense mechanism to ensure adequate clot formation for preventing fatal bleeding (24). Understanding the sequence and the magnitude of these pathophysiological processes becomes critical to define the optimal HIO dosing, in particular because the thrombocytosis may partially compensate thrombocytopenia induced by CRS and HIO (25). In this context, PK/PD models that characterize the platelet dynamics and predict the severity and the duration of the thrombocytopenia and/or thrombocytosis might be of particular clinical value in PC patients after CRS and HIO. Up to date, the effect of HIO pharmacokinetics, surgical stress response, and splenectomy on the platelet dynamics has not been characterized yet. Thus, in the current study, a previous cytokinetic model has been adapted to characterize the platelet dynamics after CRS and HIO, to evaluate the relationships between patient covariates and oxaliplatin PK/PD model parameters, and to explore the maximum tolerated HIO dose in this setting.

\section{PATIENTS AND METHODS}

Patients, Treatment, and Blood Sampling. Data from three single-arm observational studies, involving a total of 80 patients with PC, were available for the analysis. Fortyone $(51.2 \%)$ patients were treated with CRS followed by HIO diluted in isotonic 4\% icodextrin (cohort A), 21 (26.3\%) patients underwent CRS followed by HIO diluted in isotonic 5\% dextrose (Cohort B), and 18 (22.5\%) patients underwent CRS but did not receive HIO (cohort C). The details of the patient's eligibility criteria, CRS procedure, HIO treatment, study conduct, and descriptive statistics of the baseline patient characteristics have been extensively described elsewhere $(15,19,26)$. The oxaliplatin pharmacokinetics following HIO administration have been characterized in patients from cohorts $A$ and $B$ as described elsewhere $(15,19,27)$. Blood samples for the determination of platelet counts were collected before the CRS and, afterwards, daily until patient completely recovered from the hematological toxicity. Platelet counts were determined using an automated hematology analyzer
(Beckman Coulter, Inc. AcT5diff AL, Fullerton, CA, USA).

Software. Non-linear mixed-effects modeling using the first-order conditional estimation (FOCE) method implemented in NONMEM version 7.1.2 software package (ICON Development, Hanover, MD, USA) (28) was employed to conduct the model-based analysis and simulations. Compilations were achieved using gfortran compiler 4.6.2, for Windows 8.164 bits. PsN 3.4.2 was used to conduct a stratified non-parametric bootstrap. Graphical and other statistical analyses were performed using S-Plus 6.1 Professional Edition (Insightful Corporation, Seattle, WA, USA).

Pharmacokinetic and Pharmacodynamic Model Development. The schematic of the PK/PD model used to describe the dynamics of platelet counts (Fig. 1) represents a modification of the cytokinetic model proposed by Harker et al. (29) The model consisted of a precursor compartment [Prol] that represents the megakaryocyte pool, including proliferative-potential megakaryocytes, burst-forming-unit megakaryocytes, colony-forming-unit megakaryocytes, promegakaryoblasts, megakaryoblasts, mature megakaryocytes, and a platelet compartment [PLT] representing the platelets in the peripheral circulation. Consistent with the mechanism of self-renewal or mitosis, the generation of new cells in [Prol] was dependent on the number of cells in that compartment and characterized by the first-order proliferation rate constant $\left(k_{\text {prol }}\right)$, which determines the rate of cell division, together with the feedback mechanism from the circulating platelets. A feedback loop on the proliferation process was required to describe the rebound of platelets compared to its baseline value $\left(\mathrm{PLT}_{0}\right)$ and was incorporated into the model as $\left(\mathrm{PLT}_{0} / \mathrm{PLT}\right)^{\gamma}$, where $\gamma$ was the estimated parameter reflecting the increase in self-replication rate due to elevated endogenous thrombopoietin (TPO) concentrations, which are associated with platelet depletion (30). The differential equation describing the time course of megakaryocytes pool was:

$\frac{\mathrm{dProl}}{\mathrm{d} t}=k_{\text {prol }} \times$ Prol $\times\left(\frac{\mathrm{PLT}_{0}}{\mathrm{PLT}}\right)^{\gamma} \times E_{\text {Drug }}-k_{t r} \times$ Prol

where $E_{\text {Drug }}$ represents the oxaliplatin cytotoxic effect and $k_{t r}$ is the first-order rate constant that quantifies the disappearance of megakaryocyte pool from the bone marrow. From [Prol] compartment, a maturation-structured cytokinetic model was used to describe the development and maturation of platelets in blood. Similar concept has been exploited to deal with the dynamics of neutrophils and red blood cells following the administration of pegfilgrastim and erythropoietin, respectively (31,32). Briefly, platelets are represented by a series of sequential aging compartments $\left(N_{\text {PLT }}\right)$ with transition rates $k_{t r}$. Circulating platelets are removed from blood by two main mechanisms: random destruction (quantified by the first-order elimination rate constant, $k_{s}$ ) and programmed cell death (quantified by platelet lifespan, which depends on $k_{t r}$ ). The differential equations 
governing the platelet dynamics model (Eqs. 2, and 3) were

$$
\begin{aligned}
\frac{\mathrm{dPLT}_{1}}{\mathrm{~d} t}= & k_{t r} \times \eta \times \mathrm{Prol}_{-} k_{t r} \times \mathrm{PLT}_{1}-k_{s} \times \mathrm{PLT}_{1} \\
\frac{\mathrm{dPLT}_{i}}{\mathrm{~d} t}= & k_{t r} \times \mathrm{PLT}_{i-1}-k_{t r} \times \mathrm{PLT}_{i}-k_{s} \times \mathrm{PLT}_{i} \\
& \Rightarrow \text { where } i=2, \ldots, N_{\mathrm{PLT}}
\end{aligned}
$$

where $\eta$ was fixed to 4000 because each megakaryocyte releases approximately 4000 of platelets to the circulation (33). $N_{\text {PLT }}$ was arbitrarily set to 5 in order to have a large enough number of compartments that result in a smoothed distribution of platelet lifespan (34), which was calculated from the platelet amounts in each age compartment as a secondary parameter. At steady-state, before CRS, and in absence of transfusions, $\mathrm{dProl} / \mathrm{d} t$ was equal to 0 and, therefore, $k_{\text {prol }}=k_{t r}$; the amounts in each age compartment at baseline were $\mathrm{PLT}_{0} / N_{\mathrm{PLT}}$ and the amount in Prol at baseline $\left(\mathrm{Prol}_{0}\right)$ was established to be Eq. 4:

$\operatorname{Prol}_{0}=\left(1+\frac{k_{s}}{k_{t r}}\right) \times \frac{\mathrm{PLT}_{0}}{\eta \times N_{\mathrm{PLT}}}$

A total of $5(6.25 \%)$ out of 80 patients required platelet transfusions. This process was modeled as a bolus of platelet counts administered at the time of transfusion, and the remainder transfused platelet counts, TRF, were described according to Eq. 5:

$\mathrm{TRF}=\mathrm{TRF}_{0} \times e^{-k_{t} \times t}$

where the amount of platelets administered via a bolus, $\mathrm{TRF}_{0}$, and the first-order elimination rate of the infused platelets, $k_{t}$, were estimated directly from the data. The platelet counts measured (PLT) are the sum of the platelet counts in each age compartment plus the remainder amount of platelets transfused (Eq. 6):

$\mathrm{PLT}=\mathrm{PLT}_{1}+\ldots+\mathrm{PLT}_{N_{\mathrm{PLT}}}+\mathrm{TRF}$

The release of multiple inflammatory mediators after surgery stimulates megakaryocyte proliferation and differentiation which led to an increased platelet production $(35,36)$. Therefore, CRS was assumed to increase $k_{\text {prol }}$ (Eq. 7):

$k_{\text {prol }}=\left\{\begin{array}{l}k_{\text {prol }}^{0}, \text { if } t \leq t_{\mathrm{s}} \\ k_{\text {prol }}^{0} \times\left[1+\mathrm{SP}_{\text {max }} \cdot e^{-k_{p} \times\left(t-t_{\mathrm{s}}\right)}\right], \text { if } t>t_{\mathrm{s}}\end{array}\right.$

where $k_{\text {prol }}{ }^{0}$ represents the corresponding first-order rate constant before the CRS, which starts at the time of surgery $\left(t_{\mathrm{s}}\right)$; $\mathrm{SP}_{\max }$ is the maximum stimulatory effect of the surgical stress on $k_{\mathrm{prol}}$; and $k_{p}$ is the first-order rate constant driving the disappearance of the surgical stress effect on the $k_{\text {prol }}$.

The oxaliplatin plasma concentrations were assumed to inhibit the mitosis rate of proliferative cells, $k_{\text {prol }}$, according to a power function described in Eq. 8:

$E_{\text {Drug }}=1-\alpha \times C_{p}^{\beta}$

where $\alpha$ and $\beta$ were the coefficient and the exponent of the power relationship between $E_{\text {Drug }}$ and $C_{p}$, respectively, and $C_{p}$ was the oxaliplatin plasma concentration, obtained from the empirical Bayesian estimates of the individual pharmacokinetic parameters as described elsewhere $(15,19)$. In subjects receiving $\mathrm{HIO}$ with no oxaliplatin concentrations available $(n=9,11.3 \%)$, typical values of pharmacokinetic parameters were assumed to predict time course of oxaliplatin plasma concentrations. The development of the PK/PD model was performed using a sequential process as described elsewhere (37). During the model development process, linear or sigmoid functions of $C_{p}$ or the drug concentration in a hypothetical effect compartment $\left(C_{e}\right)$ were also tested to describe the $E_{\text {Drug. }}$.

Thus, the structural model parameters estimated were the system-related parameters $\left(\mathrm{PLT}_{0}, k_{t r}, k_{s}\right.$, and $\left.\gamma\right)$, the surgery-related parameters $\left(\mathrm{SP}_{\max }\right.$ and $\left.k_{p}\right)$, and the drugrelated parameters $(\alpha$ and $\beta$ ), which were all restricted to be positive $(>0)$.

Statistical Model. The interindividual variability (IIV) in the model parameters was assumed to follow a lognormal distribution, and an exponential error model was used. Residual variability in platelet counts was evaluated using an additive error model after natural logarithmic transformation of the observations and model predictions.

Model Selection Criteria. The improvement of the fit obtained for each nested model was assessed by the likelihood ratio test. The reduction in the IIV and residual variability, the precision and the correlation in parameter estimates, as well as the examination of diagnostic plots and shrinkage were used to evaluate each candidate model (38).

Covariate Analysis. The covariates selected were age, body surface area (BSA), sex, total proteins, carrier solution, and splenectomy. The effect of these covariates on model parameters was explored following the forwardinclusion $(p<0.05)$ and backward-elimination $(p<0.01)$ process. Categorical covariates were incorporated into the model as index variables, whereas continuous covariates were evaluated using power equations after centering on the median.

Model Qualification. >Four complementary methods were employed to evaluate the model developed: nonparametric bootstrap (39), normalized prediction distribution errors (NPDE) (40, 41), prediction-corrected visual predictive check (pcVPC) (42), and numerical predictive check (NPC) (43). The NPDE distribution was explored by a frequency histogram, summary statistics stratified by cohort, and scatterplots versus the population prediction and time. The pcVPC displayed the $5^{\text {th }}, 50^{\text {th }}$, and $95^{\text {th }}$ percentiles of the observed values, and the $95 \%$ 


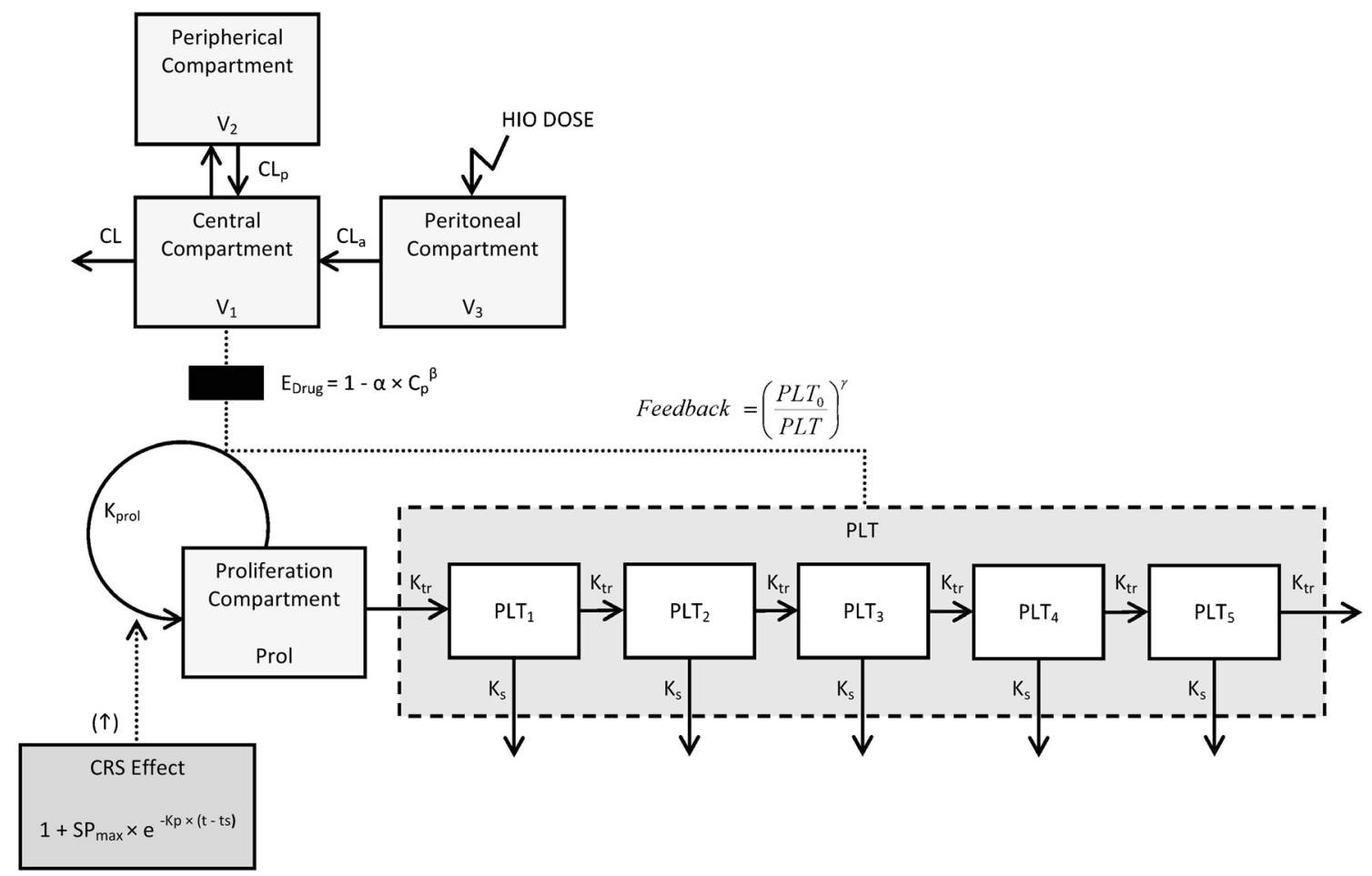

Fig. 1. Schematic of the platelet dynamics model

confidence interval for the corresponding model-based predicted percentiles computed from 1000 Monte Carlo replicates, obtained by simulating the design of the underlying dataset with the final model parameters. The NPCs were conducted for the incidence and the mean platelet counts of patients with thrombocytopenia grade $\geq 3$ (platelet counts $<50 \times 10^{9} / \mathrm{L}$ ) and thrombocytosis grade $\geq 3$ (platelet counts $>700 \times 10^{9} / \mathrm{L}$ ), stratified by cohort and the significant covariates finally included in the model.

Model-Based Simulations. A sensitivity analysis was undertaken in order to explore the surgery-related parameter effect on the platelet dynamics in patients treated with CRS and $\mathrm{HIO}$ at $200 \mathrm{mg} / \mathrm{L}$ during $30 \mathrm{~min}$. Arbitrary values of $\pm 25 \%$ and $\pm 50 \%$ variation from the estimated surgery-related model parameter value were used. Deterministic simulations were also undertaken in order to explore the role of the HIO dose (or initial oxaliplatin peritoneal concentration), treatment duration, and carrier solution on the time course of the platelet counts. Stochastic simulations were conducted to establish the relationship between dose (or initial oxaliplatin peritoneal concentrations) and the incidence of thrombocytopenia and thrombocytosis grade $\geq 3$ and determine the projected maximum tolerated HIO dose. For a range of initial oxaliplatin concentrations in the peritoneum spanning from 0 to $400 \mathrm{mg} / \mathrm{L}$, the daily platelet counts were simulated for two different HIO durations (30 and $60 \mathrm{~min}$ ), and the incidence of thrombocytopenia and thrombocytosis grade $\geq 3$ was computed for HIO treatments administered with icodextrin $4 \%$ or dextrose 5\%. For each scenario, data for 2000 virtual patients were simulated.

\section{RESULTS}

A total of 1386 platelet counts were included in the analysis dataset. The time course of the platelet counts displayed a signature pattern. The mean and the coefficient of variation $(\mathrm{CV} \%)$ of the platelet counts before CRS was $269 \times 10^{9} / \mathrm{L}(43.2 \%)$. Around 7 days after CRS, platelet counts decreased until a nadir value of $148 \times 10^{9} / \mathrm{L}(58.9 \%)$. Then, platelet counts increase up to $391 \times 10^{9} / \mathrm{L}(53.2 \%)$ approximately 25 days after the CRS. The platelet count peak in patients undergoing splenectomy, $440 \times 10^{9} / \mathrm{L}$ $(51.1 \%)$, was significantly higher than that in patients without splenectomy, $338 \times 10^{9} / \mathrm{L} \quad(55.3 \%) \quad(p=0.032)$. Finally, the platelet counts returned to the baseline values approximately 50 days after CRS.

The inclusion of a time-independent surgical stress effect on the $k_{\text {prol }}$ provided a substantial decrease in the minimum value of objective function $(\triangle \mathrm{MOFV})(\triangle \mathrm{MOFV}=-183.76)$. The response to the surgical stress is associated with the liberation of multiple inflammatory mediators, which transiently stimulate extra cell divisions in the proliferating compartment. During the resolution of inflammation, the mediators which initiated the response must somehow be removed, inactivated, or otherwise rendered impotent (44). This process was characterized by modeling the disappearance of the surgical stress effect on the proliferation process through a first-order process, quantified by $k_{p}(\Delta \mathrm{MOFV}=$ -131.98). Additional model improvement was achieved by incorporating the transfusion effect as described in methods $(\Delta \mathrm{MOFV}=-48.66)$. This three model improvements led to a $21.5 \%$ reduction in the residual variability. The surgical stress 
effect on $k_{s}$ was also tested but did not result in further model improvement. Attempts to simplify the model by assuming $k_{t r}$ equaled to $k_{s}$ lead to a substantial increased in the MOFV (44.24), the interindividual variability in model parameters (from $4.3 \%$ to $14 \%$ ) and the residual variability (5\% higher). Similarly, no improvement of the model fit was achieved by adding an effect compartment or modeling $E_{\text {Drug }}$ as a linear function of the $C_{p}$. Moreover, $E_{\text {max }}$ or sigmoid $E_{\max }$ models for $E_{\text {Drug }}$ did not converge successfully, probably because the $E_{\max }$ was not achieved within the range of $C_{p}$ evaluated and the low incidence of severe thrombocytopenia observed. Therefore, a power function of $C_{p}$ was selected to characterize the $E_{\text {Drug }}$ model.

The exploratory graphical analysis of the correlation between age, BSA, sex, total proteins, and carrier solution with PD model parameters did not suggest any statistically significant association, and, therefore, these covariates were not included in the platelet dynamics model. Nevertheless, the exploratory analysis revealed that splenectomy might have an effect on $k_{s}$, which was confirmed by the inclusion of this categorical covariate in the model ( $\Delta \mathrm{MOFV}=-6.39, d f=1, p=0.011)$. Splenectomy increased platelet lifespan from 3.23 to 7.78 days. The estimates of the final model parameters and the results of the nonparametric bootstrap analysis are presented in Table I.
The goodness-of-fit plots representing the observed platelet counts versus the population (Fig. 2a) and individual (Fig. 2b) model predictions showed a normal random scatter around the identity line, indicating the absence of significant bias. Similarly, the distribution of conditional weighted residual (45) and NPDE as a function of the population predictions (Fig. 2c, d, respectively) and time (Fig. 2e, f, respectively) did not show any trend that evidences model inadequacy or misfit. Furthermore, the NPDE distribution follows approximately a normal distribution with mean 0 and standard deviation 1 as shown in Table II. The shrinkage values (\%) for $\mathrm{PLT}_{0}, k_{t r}, k_{s}, k_{t}, k_{p}, \alpha$, and $\sigma$ were 9.79, 16.4, $26.4,77.4,33.7,40.3$, and 12.5 , respectively.

The population estimates for the final model were very similar to the mean of the $76 \%$ bootstrap replicates that minimized successfully and were contained within the $95 \%$ bootstrapped confidence intervals. From 24 bootstrap replicates that did not converged, 20 were due to rounding errors and 4 of them were terminated due to proximity of the last iteration at which the objective function was infinite. The distribution of the parameter estimates across bootstrap replicates was similar between the replicates that minimized successfully and those that did not with a relative difference lower than $8 \%$. This suggests that the covariance step failure could be related to the use of FOCE method, rather than

Table I. Parameter Estimates (Relative Standard Errors) and Non-parametric Bootstrap Analysis of the Platelet Dynamics Model

\begin{tabular}{|c|c|c|c|}
\hline \multirow[b]{2}{*}{ Platelet dynamics model parameters } & \multirow{2}{*}{$\frac{\text { Original dataset }}{\text { Estimate }^{a, b}}$} & \multicolumn{2}{|c|}{ Non-parametric bootstrap $(N=76$ replicates out of 100$)$} \\
\hline & & Mean (RSE, \%) & $95 \%$ confidence interval \\
\hline \multicolumn{4}{|l|}{ System-related parameters } \\
\hline $\mathrm{PTL}_{0}\left(\times 10^{9} / \mathrm{L}\right)$ & 237 & $236(3.72)$ & $222-253$ \\
\hline$k_{t r}\left(\times 10^{-3} / \mathrm{h}\right)$ & 7.09 & $8.09(35.5)$ & $1.96-13.3$ \\
\hline$k_{s}\left(\times 10^{-3} / \mathrm{h}\right)$ & 8.86 & $8.18(16.8)$ & $6.00-10.6$ \\
\hline Splenectomy factor $(\Phi)$ & 0.475 & $0.557(26.2)$ & $0.324-0.900$ \\
\hline$\gamma$ & 0.621 & $0.612(45.9)$ & $0.266-1.181$ \\
\hline \multicolumn{4}{|l|}{ Transfusion-related parameters } \\
\hline $\mathrm{TRF}_{0}\left(\times 10^{9} / \mathrm{L}\right)$ & 255 & $248(29.2)$ & $102-336$ \\
\hline$k_{t}(/ \mathrm{h})$ & 0.104 & $0.109(53.8)$ & $0.049-0.170$ \\
\hline \multicolumn{4}{|l|}{ Surgical-related parameters } \\
\hline $\mathrm{SP}_{\max }$ & 2.09 & $2.06(49.6)$ & $1.54-3.27$ \\
\hline$k_{p}\left(\times 10^{-3} / \mathrm{h}\right)$ & 3.43 & $3.66(44.9)$ & $2.17-8.06$ \\
\hline \multicolumn{4}{|l|}{ Drug-related parameter } \\
\hline$\alpha(\mathrm{L} / \mathrm{mg})$ & 0.881 & $0.903(17.2)$ & $0.648-1.189$ \\
\hline$\beta$ & 2.63 & $2.37(17.4)$ & $1.59-3.24$ \\
\hline \multicolumn{4}{|l|}{ Interindividual variability $(\mathrm{CV} \%)$} \\
\hline$\omega \mathrm{PLT}_{0}$ & 32.9 & $32.6(20.9)$ & $26.3-39.2$ \\
\hline$\omega k_{t r}$ & 47.1 & $46.9(44.6)$ & $26.7-70.7$ \\
\hline$\omega k_{s}$ & 80.0 & $81.1(42.8)$ & $33.3-107$ \\
\hline$\omega k_{t}$ & 62.8 & $60.1(48.8)$ & $12.8-88.4$ \\
\hline$\omega k_{p}$ & 85.6 & $98.8(60.5)$ & $38.6-145$ \\
\hline$\omega \alpha$ & 56.9 & $61.9(31.1)$ & $45.0-77.1$ \\
\hline \multicolumn{4}{|l|}{ Residual variability (CV\%) } \\
\hline$\varepsilon$ & 25.5 & $25.6(9.98)$ & $20.1-30.0$ \\
\hline
\end{tabular}

$P L T_{0}$ baseline value of absolute platelet count, $k_{t r}$ platelet maturation rate constant, $k_{s}$ platelet random destruction rate constant, $\gamma$ feedback effect on progenitor cell proliferation, $T R F_{0}$ amount of transfused platelets administered via a bolus, $k_{t}$ first-order elimination rate of the transfused platelets, $\phi$ splenectomy effect in $k_{s}, S P_{\max }$ maximum surgical stimulatory effect in $k_{\text {prol }}, k_{p}$ first-order rate constant driving the disappearance of the surgical effect in $k_{\text {prol }}, \alpha$ coefficient of the power relationship between the drug effect $\left(E_{\text {drug }}\right)$ and the oxaliplatin peritoneal concentrations $\left(C_{p}\right), \beta$ exponent of the power relationship between $E_{\mathrm{drug}}$ and $C_{p}, \varepsilon$ residual variability

${ }^{a}$ The shrinkage values $(\%)$ for $\mathrm{PLT}_{0}, k_{t r}, k_{s}, k_{t}, k_{p}, \alpha$, and $\sigma$ were 9.79, 16.4, 26.4, 77.4, 33.7, 40.3, and 12.5, respectively (38)

${ }^{b}$ The covariance step failed. Therefore, RSE of platelet dynamics model parameters were obtained from the bootstrap analysis 
a

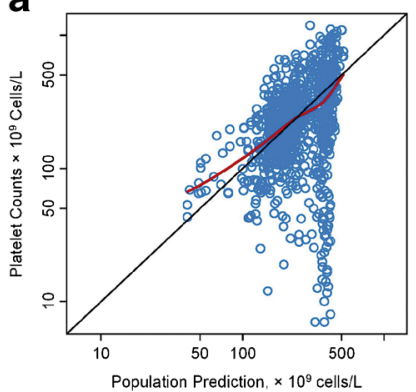

C

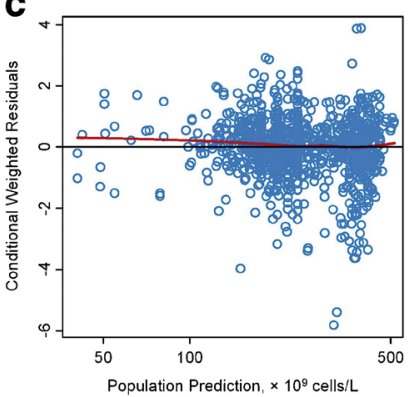

e

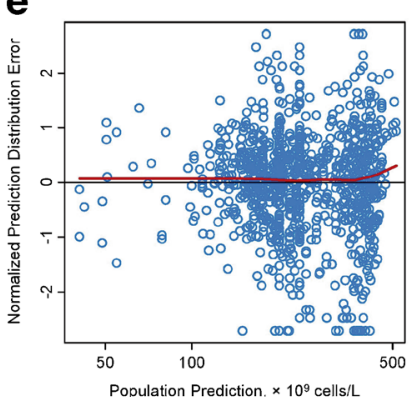

b

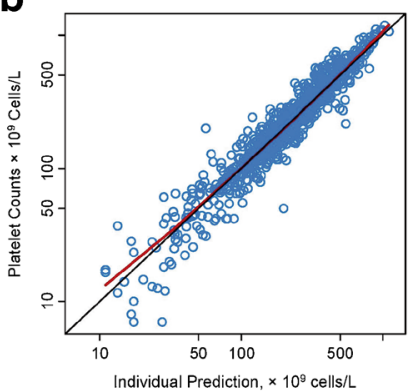

d

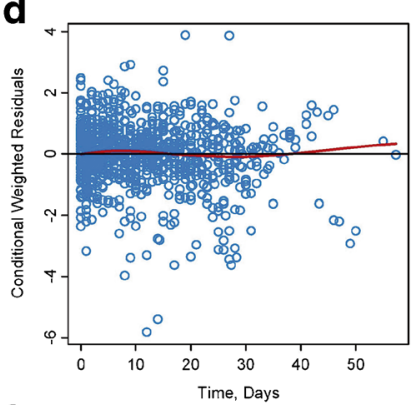

$f$

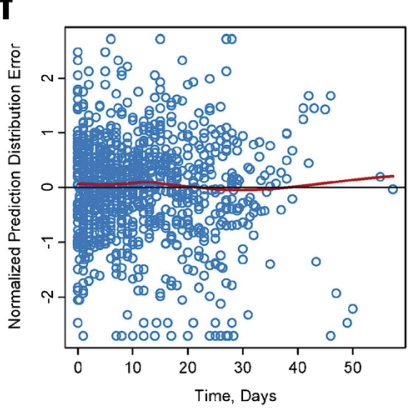

Fig. 2. Goodness-of-fit plots for the platelet dynamics model

model over-parameterization or instability. The precision of the parameter estimates for the fixed effects was acceptable with relative standard errors (RSE) lower than $50 \%$, except for $k_{t}$ which was $53.8 \%$. This RSE was slightly higher because only $6.25 \%$ of the patients received a platelet transfusion. In addition, the precision for the random effect was also adequate with RSE ranging from $20.9 \%$ to $48.8 \%$, except for $\omega k_{p}$. The precision for the $\omega k_{p}$ was $60.5 \%$ is not surprising since the estimation of $k_{p}$ is really low $\left(3.43 \times 10^{-3} / \mathrm{h}\right)$ and therefore it is very difficult to estimate the individual parameter precisely. Furthermore, the exclusion of the variability term in $k_{p}$ resulted in an increase of MOFV (54.09), an increase in the other interindividual variability parameters ranging from $1.9 \%$ to $14.7 \%$ and a $3.8 \%$ increase in the residual variability. Therefore, the removal of this variability term was not finally considered.

The pcVPC (Fig. 3, upper panels) evidenced that the model developed was appropriate to describe the time course

Table II. Numerical Predictive Check of the Incidence of Thrombocytopenia Grade $\geq 3$ and Thrombocytosis Grade $\geq 3$ and Its Mean Platelet Counts, and NPDE Mean and Standard Deviation Stratified by Cohort

\begin{tabular}{|c|c|c|c|c|c|c|c|}
\hline & & \multicolumn{2}{|l|}{ Cohort A } & \multicolumn{2}{|l|}{ Cohort B } & \multicolumn{2}{|l|}{ Cohort C } \\
\hline \multicolumn{2}{|c|}{ Numerical predictive check } & Observed & Predicted $(95 \% \mathrm{CI})$ & Observed & Predicted $(95 \% \mathrm{CI})$ & Observed & Predicted $(95 \% \mathrm{CI})$ \\
\hline \multirow{2}{*}{$\begin{array}{l}\text { Thrombocytopenia } \\
\quad \text { grade } \geq 3\end{array}$} & Incidence (\%) & 19.5 & $12.2(4.88$ to 19.5$)$ & 9.52 & $9.52(0.00$ to 19.1$)$ & 5.56 & $0.00(0.00$ to 5.56$)$ \\
\hline & $\begin{array}{l}\text { Mean platelet counts } \\
\quad\left(\times 10^{9} \text { cells } / \mathrm{L}\right)\end{array}$ & 36.0 & $32.9(15.1$ to 45.4$)$ & 45.1 & $31.1(7.78$ to 46.2$)$ & 42.0 & 43.8 (26.5 to 49.3$)$ \\
\hline \multirow{2}{*}{$\begin{array}{l}\text { Thrombocytosis } \\
\text { grade } \geq 3\end{array}$} & Incidence $(\%)$ & 9.76 & $14.6(7.32$ to 26.8$)$ & 28.6 & $23.8(9.52$ to 38.1$)$ & 11.1 & $22.2(11.1$ to 38.9$)$ \\
\hline & $\begin{array}{l}\text { Mean platelet counts } \\
\left(\times 10^{9} \text { cells } / \mathrm{L}\right)\end{array}$ & 773 & 822 (742 to 1002$)$ & 846 & $839(758$ to 1041$)$ & 761 & $842(739$ to 1123$)$ \\
\hline \multirow[t]{2}{*}{ NPDE } & Mean & -0.07 & $-0.06(-0.14$ to 0.01$)$ & 0.07 & $0.06(-0.03$ to 0.15$)$ & 0.03 & $0.02(-0.08$ to 0.13$)$ \\
\hline & Standard deviation & 0.98 & $0.98(0.91$ to 1.05$)$ & 0.96 & $0.95(0.89$ to 1.01$)$ & 0.98 & $0.96(0.88$ to 1.07$)$ \\
\hline
\end{tabular}



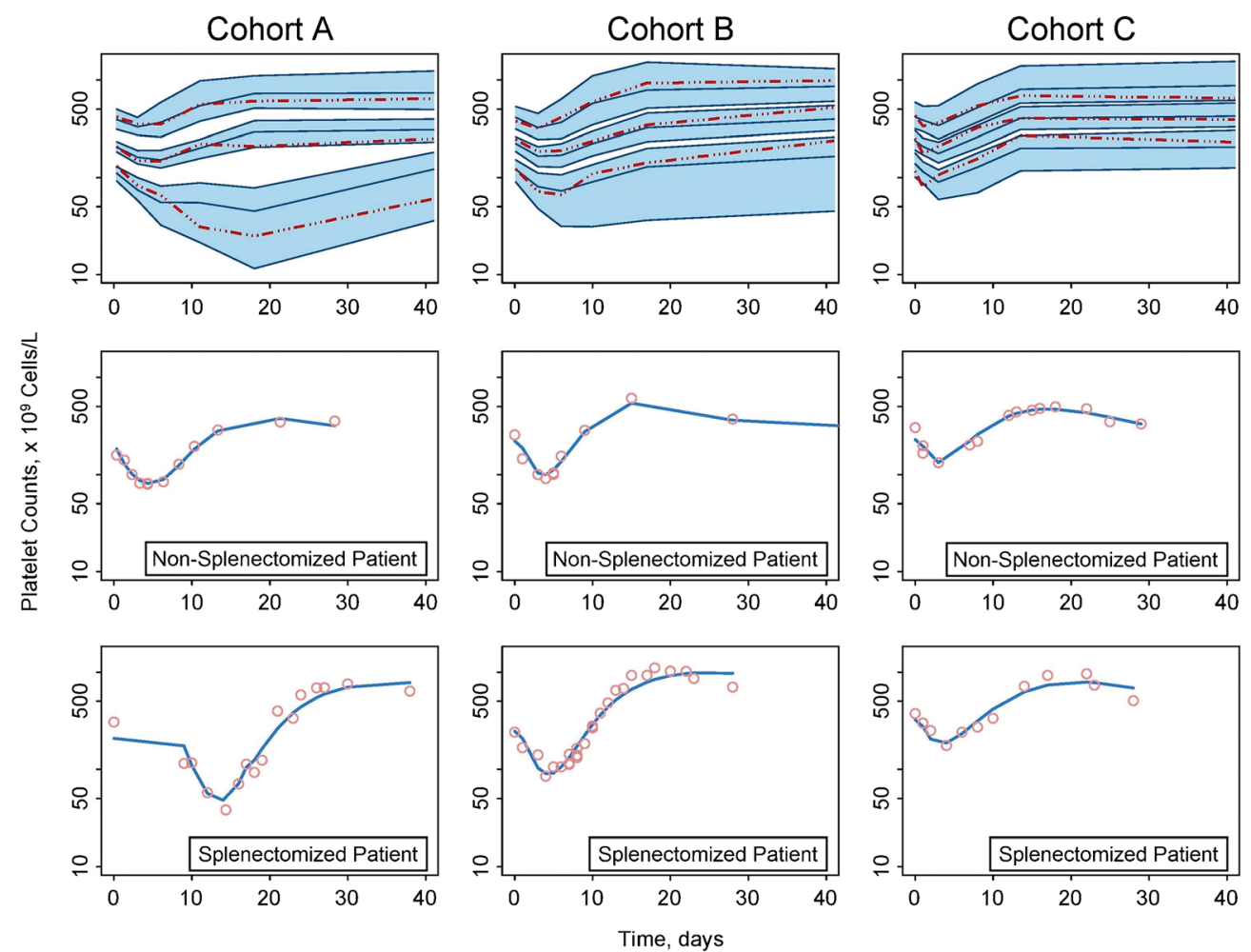

Fig. 3. Prediction-corrected visual predictive check (upper panels), and representative individual time course of platelet counts and the corresponding model predictions for non-splenectomized (middle panels) and splenectomized patients (lower panels) stratified by cohort

of thrombocytopenia and subsequent thrombocytosis, and its variability in PC patients after CRS, with or without $\mathrm{HIO}$ administration, regardless of the carrier solution used (42). Figure 3 shows the model ability to describe the individual time course of platelets for non-splenectomized (middle panels) and splenectomized (lower panels) patients randomly selected from the three cohorts of patients analyzed in this study. The NPC (Table II) qualifies the model to properly describe the incidence of thrombocytopenia and thrombocytosis grade $\geq 3$, as well as the mean platelets counts in subjects with these toxicities in the three cohorts studied.

The PK/PD model properly characterizes the transient thrombocytopenia observed immediately after the CRS and HIO, the rebound phenomena characterized by a transient thrombocytosis achieved around 25 days after the CRS, and the subsequent return to baseline of the platelet counts (Fig. 4). Each part of the platelet time course signature pattern is governed by different model parameters. The initial transient thrombocytopenia is due to the HIO effect in the bone marrow. During the first days after CRS, the elevated endogenous TPO concentration drives the feedback process, which contributes to further stimulate the megakaryocyte proliferation and differentiation. Around 7 days after the CRS, the platelet counts start to increase because the CRS effect becomes larger than the HIO effect on $k_{\text {prol }}$, which ultimately leads to a delayed increase in platelet counts and thrombocytosis. The thrombocytosis severity is mainly determined by the parameter $\mathrm{SP}_{\max }$ (Fig. 4a). The effect of $k_{p}$ in platelet count profile is similar, but of opposite magnitude, than that described by $\mathrm{SP}_{\max }$. Thus, higher values of $k_{p}$ are related to a rapid attenuation of CRS effect on $k_{\text {prol }}$, which leads the platelet counts to return to baseline values earlier (Fig. 4c). The effect of the two surgery-related parameters on the shape of the platelet count profile in patients who underwent splenectomy (Fig. 4b-d) is similar to the effect observed in patients who did not have splenectomy. However, for a given change in the surgery-specific parameter, the magnitude of the difference in the platelet count profile is larger in splenectomized patients, relative to non-splenectomized patients. This phenomenon is due to the $47.5 \%$ reduction in $k_{s}$ (or 2.1-fold increase in platelet lifespan) in splenectomized patients, which leads to a lower platelet nadir, higher platelet peak, and longer time to reach platelet nadir and peak in splenectomized patients, relative to non-splenectomized patients. These results are consistent with the physiological role of the spleen, which governs the platelet senescence and random removal from the circulation.

Deterministic simulations clearly show that the main determinants of the thrombocytopenia severity and duration are the initial HIO concentration in the peritoneum, the treatment duration, and the splenectomy procedure (Fig. 5). The carrier solution effect was negligible when describing the thrombocytopenia severity and the duration caused by HIO. For example, the nadir of platelet counts in non-splenectomized patients following CRS and 30-min HIO administration of 100, 200 , and $400 \mathrm{mg} / \mathrm{L}$ with dextrose $5 \%$ was $156,99.9$, and $55.4 \times$ $10^{9} / \mathrm{L}$, respectively. If the HIO duration was maintained during $60-\mathrm{min}$, these values were reduced to $122,26.9$, and $8.24 \times 10^{9} / \mathrm{L}$, respectively. The nadir values determined in the splenectomized patients were higher than those in non-splenectomized patients. For example, the nadir of platelet counts in splenectomized patients after CRS and 30-min HIO administration of 100, 200, and $400 \mathrm{mg} / \mathrm{L}$ with dextrose $5 \%$ was 192,137 , and $84.7 \times 10^{9} / \mathrm{L}$, 

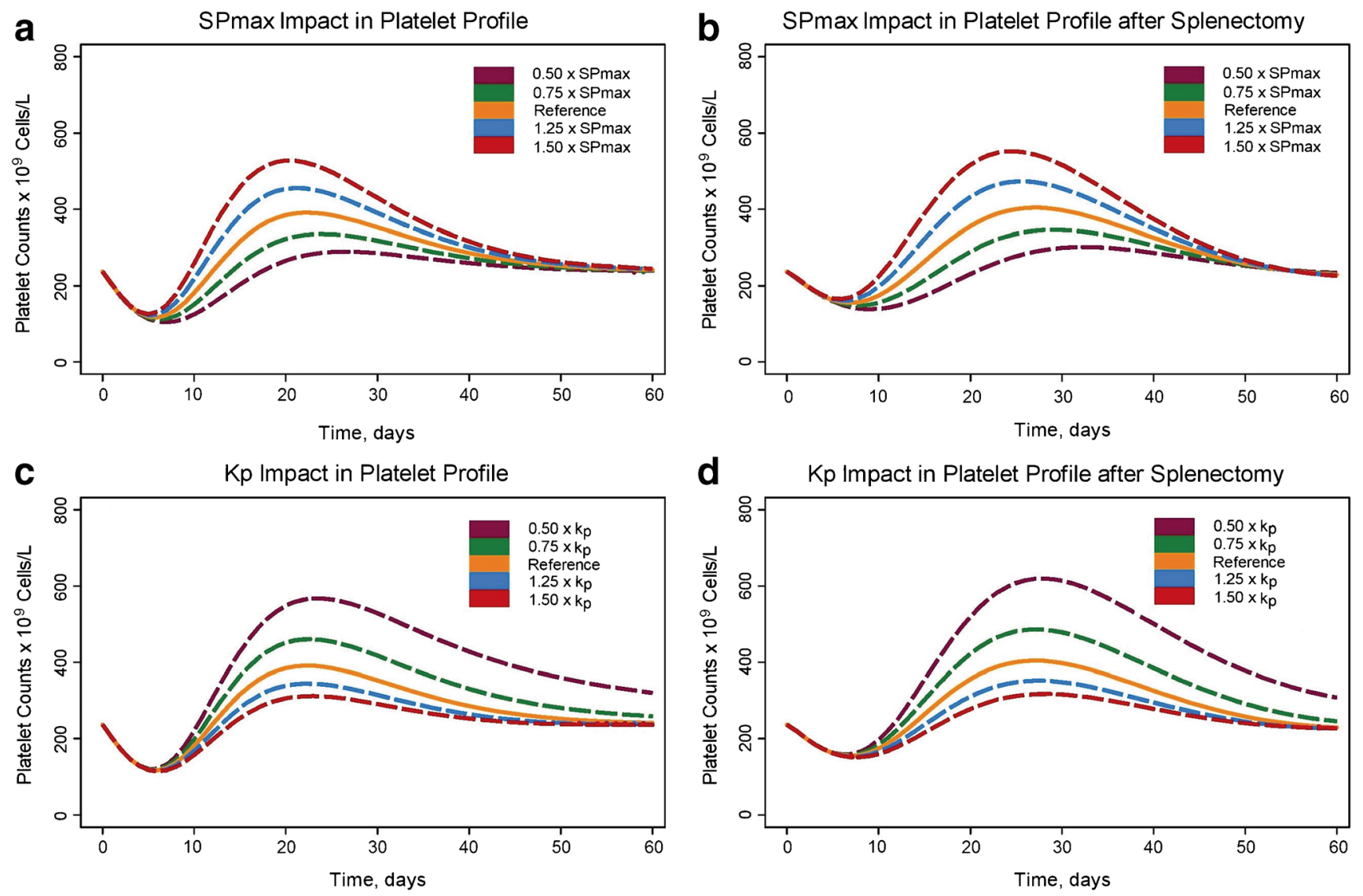

Fig. 4. Influence of surgery-specific parameters $\mathrm{SP}_{\max }$ and $k_{p}$ on platelet profiles

respectively. If the HIO duration was prolonged to 60-min, these values decreased to $159,44.7$, and $14.7 \times 10^{9} / \mathrm{L}$, respectively. These results are encouraging because probably higher HIO doses could be used in splenectomized patients without increasing the risk of hematological toxicity, which is reversible and short-lasting.

A $30-\mathrm{min}$ HIO administration of 270 or $320 \mathrm{mg} / \mathrm{L}$ diluted in dextrose $5 \%$ leads to approximately $33 \%$ incidence of thrombocytopenia grade $\geq 3$ in splenectomized or nonsplenectomized patients, respectively (Fig. 6, upper panels). If the treatment duration is extended to $60-\mathrm{min}$, the initial HIO concentration should be reduced by $60 \%$ to achieve similar incidence of thrombocytopenia grade $\geq 3$, regardless of the splenectomy. Moreover, if instead of using dextrose $5 \%$ as carrier solution, an isotonic icodextrin $4 \%$ solution is employed, the initial HIO concentrations should be increased by $10 \%$ or $12 \%$ for a HIO duration treatment of 30 or $60 \mathrm{~min}$, respectively, in order to obtain similar incidence to that obtained with dextrose $5 \%$, regardless of the presence or absence of the splenectomy.

While the HIO duration and the carrier solution have a minor impact on the incidence of thrombocytosis grade $\geq 3$ (Fig. 6, lower panels), the presence of splenectomy and the initial oxaliplatin concentration in the peritoneum determine this toxicity. The splenectomized patients had a slightly higher risk of severe thrombocytosis than those who did not undergo splenectomy. Taking as reference a 30min HIO administered with dextrose $5 \%$, the absolute difference in the incidence of thrombocytosis grade $\geq 3$ due to the splenectomy ranges from $2.0 \%$ to $10.1 \%$ across the oxaliplatin concentrations in the peritoneum evaluated. If the initial HIO concentration in the peritoneum increased from 0 to $500 \mathrm{mg} / \mathrm{L}$, the incidence of thrombocytosis grade $\geq 3$ will decrease by $4.0 \%$ or $9.6 \%$ in splenectomized or nonsplenectomized patients, respectively.

\section{DISCUSSION}

The majority of the side effects of CRS with or without $\mathrm{HIO}$ are related to the surgical tumor debulking procedure itself. The most common complications following CRS are postoperative hemorrhage, blood clot formation, anastomotic dehiscence, intestinal perforation, wound complications and infections, among others. The combination of HIO with CRS comes with an increased risk of the surgical complications and chemotherapy induced toxicity. These may include hematological toxicity, peripheral neuropathy, nephrotoxicty, nausea, and vomiting which are related to the antineoplastic nature of oxaliplatin. Except the hematological toxicities, the toxicities observed in the cohorts investigated in the current analysis were very similar to those observed in the literature, and there were no major differences in toxicity or post morbidity complications between cohorts analyzed. However, this finding should be considered with caution because the small sample size across cohorts precludes any formal statistical comparison.

Thrombocytopenia and neutropenia are the main doselimiting hematological toxicities related with oxaliplatin 

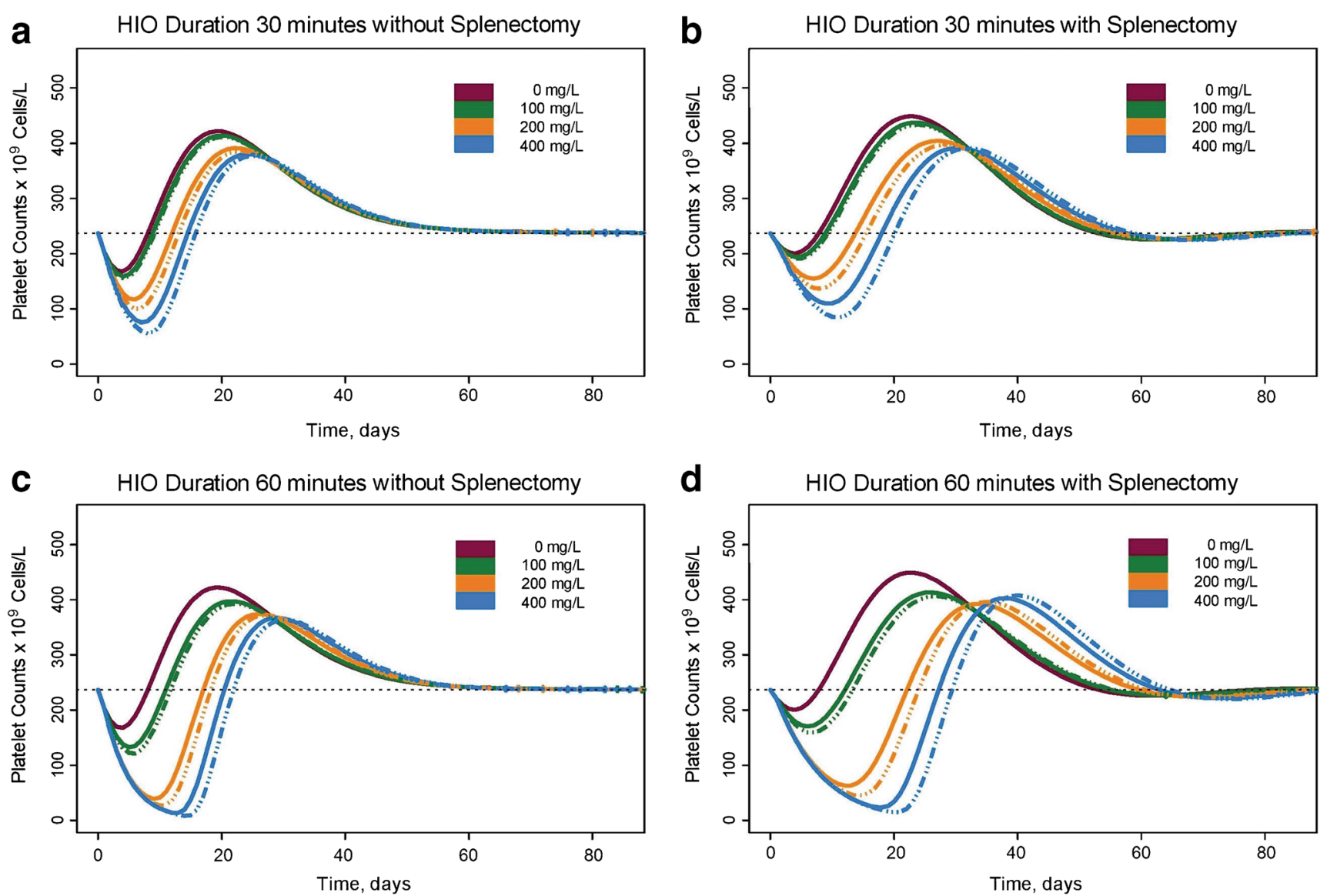

Fig. 5. Effect of initial oxaliplatin concentrations on the peritoneum, treatment duration, and carrier solution on platelet dynamics in nonsplenectomized and splenectomized patients. The solid lines represent HIO diluted in icodextrin $4 \%$ while the dashed lines represent HIO diluted in dextrose $5 \%$

intravenous treatment. Since the evaluation of neutropenia during the treatment with HIO after CRS in carcinomatosis patients has been previously described (19), we now aimed to describe the relationship between HIO pharmacokinetics and the time course of the platelet counts in the same target population for the first time. In this context, we have extended the cytokinetic model previously developed by Harker et al. (29), to properly characterize the transient thrombocytopenia and subsequent thrombocytosis induced by CRS and HIO treatment.

Under normal circumstances, platelet follows an orderly maturation and progression from the bone marrow to the circulatory system; however, both, CRS and HIO modify the normal homeostasis of platelets. HIO induces an acuteimmediate thrombocytopenia due to the oxaliplatin myelosuppressive effect, while CRS leads to a postoperative thrombocytosis response due to an increased platelet production $(19,40)$. These processes have been successfully accounted for in the PK/PD model and allows to get better understanding of the platelet dynamics in patients with CRS with or without HIO.

A few hours after the HIO administration, the platelet counts start to decline reaching a nadir at a median of the 7 days after CRS, similar to the value reported by Canda et al. (46). Oxaliplatin suppresses bone marrow function by a direct apoptotic effect on the megakaryocytic progenitors (21). The HIO-induced thrombocytopenia may potentially lead to acute bleeding events during the first days after the CRS and HIO treatment. The relationship between $C_{p}$ and the bone marrow suppression was quantified in the PKPD model by a power function (Table I), suggesting that oxaliplatin thrombocytopenic effects is about 13 -fold more potent than its neutropenic effect (19).

Two different mechanisms may explain the platelet count rebound subsequent to the platelet nadir. Firstly, a feedback mechanism on $k_{\text {prol }}$ accounts for the physiological response to an acute decrease in platelet counts and c-mpl receptors caused by HIO (47). Since fewer TPO binding sites are available due to the low number of platelet counts induced by HIO, TPO increases and stimulates the proliferation of precursor cell, which ultimately will result in thrombocytosis (48). The magnitude of this feedback mechanism (0.621) is higher than the values reported in the literature for other anticancer agents (range 0.135 to 0.233$)(49,52)$, probably because, after a major surgery, there is a further increase in circulating TPO due to its release from activated platelets (35).

Secondly, CRS was also assumed to increase $k_{\text {prol }}$ because the surgical stress is associated with the liberation of multiple inflammatory mediators like leukotriene B4, complement component C5a, interleukin-16, $\mathrm{TNF}_{\alpha}$, and $\mathrm{G}-$ CSF, which upregulate precursor cell proliferation $(36,50)$. These cytokines were able to increase by 2.09 -fold the megakaryocyte proliferation rate, which is close to the value 


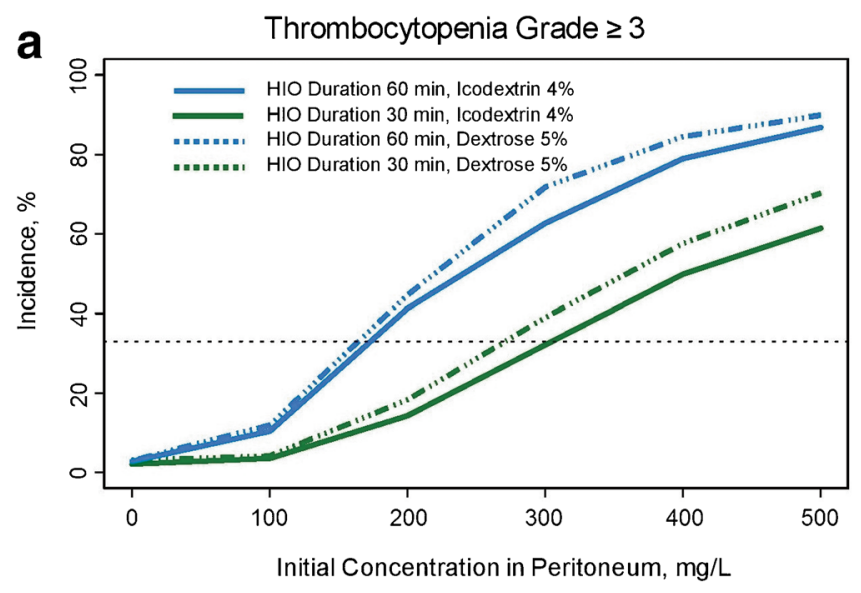

C

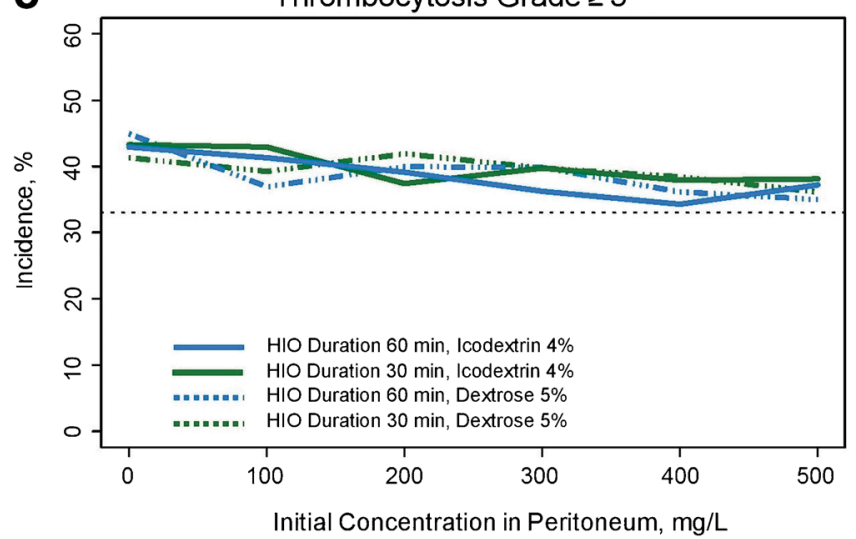

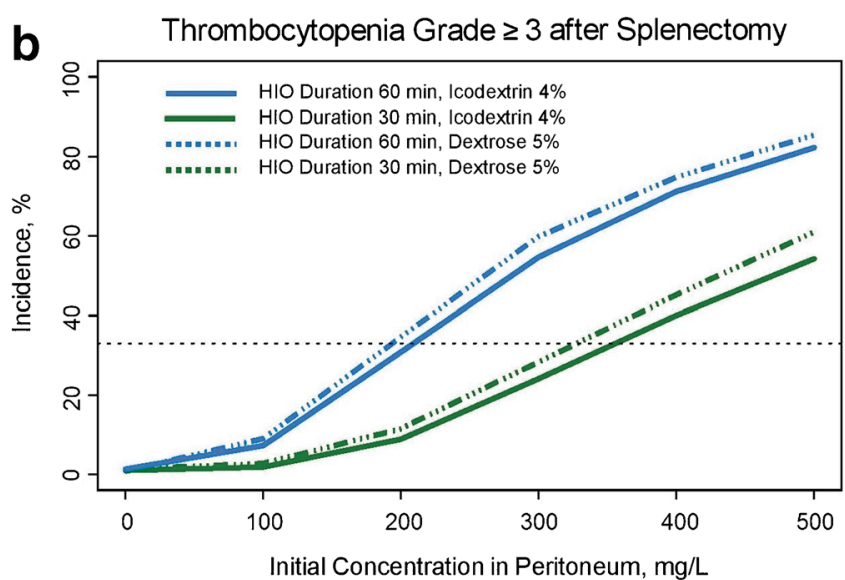

d

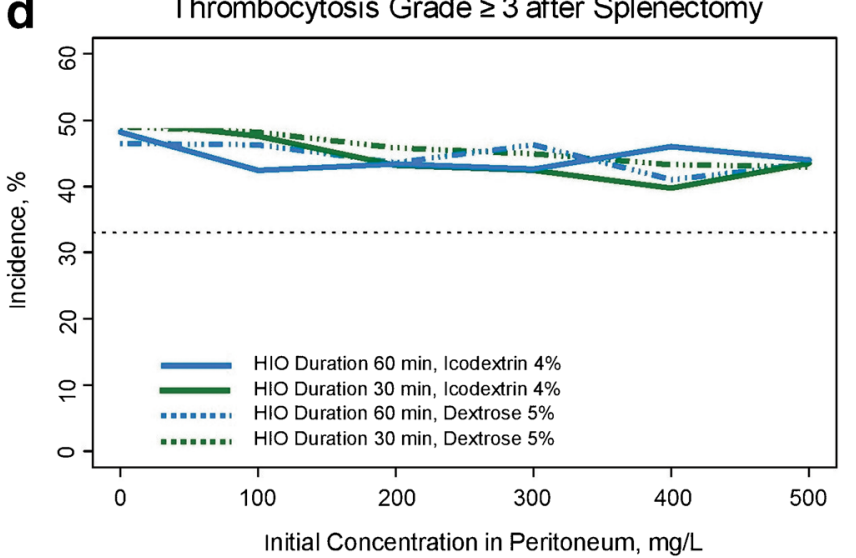

Fig. 6. Effect of initial oxaliplatin concentrations in the peritoneum, treatment duration, and carrier solution in the incidence of thrombocytopenia and thrombocytosis in non-splenectomized and splenectomized patients

reported by Drayer et al. (3.1- to 5.2-fold increase) based on an in vitro analysis (51). Both, the feedback mechanism and the surgical stress contributed to a slow delayed increase in platelet production, which became apparent at least, 7 days after CRS and leads to platelet count peaks around the 25th day after CRS. Subsequently, platelet counts return to baseline values by day 50 (48), which is consistent with the half-life (8.42 days) associated to the attenuation of the surgery effect on precursor cells.

With respect to other parameters, the estimation of $\mathrm{PLT}_{0}$ before CRS was $237 \times 10^{9} / \mathrm{L}(32.9 \%)$ which is consistent with physiologic platelet values $(52,53)$. The total amount of platelet transfused to these patients resulted in an increase in platelet counts of $255 \times 10^{9} / \mathrm{L}$, which were eliminated from systemic circulation with a relatively short half-life of $6.66 \mathrm{~h}$ and, in some cases, require additional transfusion within 34 days, if thrombocytopenia still persists. This finding should be interpreted with caution given the limited number of patients who received transfusions.

A total of $37.5 \%$ of the patients were splenectomized and, on average, had a 2.1-fold increase in platelet counts relative to non-splenectomized patients (Fig. 3). Literature data suggest that splenectomy is associated with a 1.3- to 2.6fold increase in platelet counts in the postoperative period $(54,55)$. An estimated $47.5 \%$ reduction in $k_{s}$ suggested that the platelet lifespan increased from 3.23 days in nonsplenectomized patients to 7.78 days in splenectomized patients. The estimates of platelet lifespan in nonsplenectomized patients were lower than values previously reported, which ranged from 6 to 9 days in the absence of any surgical procedure $(29,49,56)$. This difference is probably due to the increase in platelet destruction and consumption associated with the aggressive CRS treatment.

The four complementary methods used to evaluate the predictive performance of the platelet dynamics model showed the precision of the NONMEM parameter estimates was acceptable and the model developed was suitable to describe the thrombocytopenia and thrombocytosis events caused by CRS and HIO, as well as the time course of platelet counts and its associated variability. This successful model qualification suggests that the severity and duration of thrombocytopenia and subsequent thrombocytosis can be predicted in PC patients treated with CRS and HIO. Therefore, simulations were undertaken to explore the relationship between the HIO dose and the incidence of severe thrombocytopenia and thrombocytosis in both splenectomized and non-splenectomized patients, stratified by the treatment duration and carrier solution.

Deterministic simulations revealed that HIO induced a reversible and short-lasting thrombocytopenia, which is largely dependent on the dose administered (or the initial HIO concentration in the peritoneum) and the treatment duration. Figure 5 shows that increasing the initial HIO concentration in the peritoneum and/or extending the $\mathrm{HIO}$ 
duration leads to a greater fluctuation in platelet counts and, consequently, increases the likelihood of severe thrombocytopenia. Model-based stochastic simulations also indicated that it is possible to reduce the thrombocytopenia incidence and severity by using treatment regimens with shorter HIO durations, while the overall dose administered (and the initial $\mathrm{HIO}$ concentration in the peritoneum) remains the same.

As previously described, icodextrin $4 \%$ is an isotonic high molecular weight solution widely used for peritoneal dialysis that has also been employed as carrier solution for HIO. Isotonic high molecular weight solutions, such as icodextrin, are not absorbed, maintain the intraperitoneal fluid volume longer, and reduced the amount of oxaliplatin absorbed from the peritoneum cavity into the plasma. Consequently, icodextrin $4 \%$ provides a slight reduction in the incidence and severity of thrombocytopenia with respect to other isotonic carrier solutions like dextrose $5 \%$ $(14,56)$. However, for a given HIO dose and carrier solution (dextrose $5 \%$ or icodextrin $4 \%$ ), extending the duration from 30 to $60 \mathrm{~min}$ was associated with a 1.5- to 3.4-fold increase in the risk of thrombocytopenia grade $\geq 3$. As a consequence of the nonlinear drug effect, an approximate $32.5 \%$ or $80.4 \%$ decrease in the platelet counts at the $10^{\text {th }}$ day after CRS is predicted per each $100 \mathrm{mg} / \mathrm{L}$ increase in the initial HIO concentration for 30 or $60 \mathrm{~min}$ of treatment, regardless of the carrier solution.

Qualitatively, the results of the simulation exercise emphasized the relative importance of the HIO dose, treatment duration, carrier solution, as well as splenectomy effect on the incidence of severe thrombocytopenia. However, generalization of these quantitative results should be done with caution, due to the semi-mechanistic nature of the platelet dynamics model developed, the range of doses evaluated, the limited number of patients, and the specific characteristics of this complex treatment in our center. These circumstances per se demand further model evaluation in additional studies.

In summary, the time course of platelet counts was well characterized by the model developed, which simultaneously accounts for the acute-immediate thrombocytopenia response induced by the CRS and the HIO effects in bone marrow, as well as the subsequent thrombocytosis due to the natural defense mechanism to prevent major bleedings. This model suggests that, following CRS and HIO, thrombocytopenia and thrombocytosis were reversible and short-lasting. The severity of the thrombocytopenia and thrombocytosis were inversely correlated, with splenectomized patients having thrombocytopenia of lower severity and thrombocytosis of higher severity. The HIO dose and treatment duration determine the severity and duration of the thrombocytopenia, which is the HIO dose-limiting toxicity. Relative to the HIO dosing regimens used up to date, higher HIO dose or longer duration of HIO treatment could be used to treat PC patients, especially in splenectomized patients, without substantially increasing the risk of major hematological toxicity.

\section{ACKNOWLEDGMENTS}

The authors would like to thank the patients and medical, nursing, and laboratory staff of the Hospital Quirón Torrevieja who participated in the present study. This work was supported by Consellería de Sanidad of Comunidad Valenciana (Grant GE-079/11).

\section{COMPLIANCE WITH ETHICAL STANDARDS}

Conflict of Interest The authors declare that they have no competing interests.

Disclaimer The views expressed in this article are the personal view of the authors reflecting their scientific knowledge of this topic and should not be understood or quoted as being made on behalf of the companies or institutions where the authors work.

\section{REFERENCES}

1. Brücher BL, Piso P, Verwaal V, et al. Peritoneal carcinomatosis: cytoreductive surgery and HIPEC-overview and basics. Cancer Investig. 2012;30:209-24.

2. Mohamed F, Cecil T, Moran B, et al. A new standard of care for the management of peritoneal surface malignancy. Curr Oncol. 2011;18:84-96.

3. de Cuba EM, Kwakman R, Knol DL, et al. Cytoreductive surgery and HIPEC for peritoneal metastases combined with curative treatment of colorectal liver metastases: systematic review of all literature and meta-analysis of observational studies. Cancer Treat Rev. 2013;39:321-7.

4. Hompes D, D'Hoore A, Van Cutsem E, et al. The treatment of peritoneal carcinomatosis of colorectal cancer with complete cytoreductive surgery and hyperthermic intraperitoneal perioperative chemotherapy (HIPEC) with oxaliplatin: a Belgian multicentre prospective phase II clinical study. Ann Surg Oncol. 2012;19:2186-94.

5. Mulier S, Claes JP, Dierieck V, et al. Survival benefit of adding hyperthermic intraperitoneal chemotherapy (HIPEC) at the different time-points of treatment of ovarian cancer: review of evidence. Curr Pharm Des. 2012;18:3793-803.

6. Pomel C, Ferron G, Lorimier G, et al. Hyperthermic intraperitoneal chemotherapy using oxaliplatin as consolidation therapy for advanced epithelial ovarian carcinoma. Results of a phase II prospective multicentre trial. CHIPOVAC study. Eur J Surg Oncol. 2010;36:589-93.

7. Yang XJ, Huang CQ, Suo T, et al. Cytoreductive surgery and hyperthermic intraperitoneal chemotherapy improves survival of patients with peritoneal carcinomatosis from gastric cancer: final results of a phase III randomized clinical trial. Ann Surg Oncol. 2011;18:1575-81.

8. Ströhlein MA, Bulian DR, Heiss MM. Clinical efficacy of cytoreductive surgery and hyperthermic chemotherapy in peritoneal carcinomatosis from gastric cancer. Expert Rev Anticancer Ther. 2011;11:1505-8.

9. Sugarbaker PH. Intraperitoneal chemotherapy and cytoreductive surgery for the prevention and treatment of peritoneal carcinomatosis and sarcomatosis. Semin Surg Oncol. 1998;14:254-61.

10. Elias D, Pocard M, Goere D. HIPEC with oxaliplatin in the treatment of peritoneal carcinomatosis of colorectal origin. Cancer Treat Res. 2007;134:303-18.

11. Alcindor T, Beauger N. Oxaliplatin: a review in the era of molecularly targeted therapy. Curr Oncol. 2011;18:18-25.

12. Elias D, El Otmany A, Bonnay M, et al. Heated intraoperative intraperitoneal oxaliplatin after complete resection of peritoneal carcinomatosis: pharmacokinetics and tissue distribution. Ann Oncol. 2002;13:267-72.

13. Atallah D, Marsaud V, Radanyi C, et al. Thermal enhancement of oxaliplatin-induced inhibition of cell proliferation and cell cycle progression in human carcinoma cell lines. Int J Hyperth. 2004:20:405-19.

14. Ferron G, Dattez S, Gladieff L, et al. Pharmacokinetics of heated intraperitoneal oxaliplatin. Cancer Chemother Pharmacol. 2008;62:679-83. 
15. Pérez-Ruixo C, Valenzuela B, Peris JE, et al. Population pharmacokinetics of hyperthermic intraperitoneal oxaliplatin in patients with peritoneal carcinomatosis after cytoreductive surgery. Cancer Chemother Pharmacol. 2013;71:693-704.

16. Elias D, Sideris L. Pharmacokinetics of heated intraoperative intraperitoneal oxaliplatin after complete resection of peritoneal carcinomatosis. Surg Oncol Clin N Am. 2003;12:755-69.

17. Massari C, Brienza S, Rotarski M, et al. Pharmacokinetics of oxaliplatin in patients with normal versus impaired renal function. Cancer Chemother Pharmacol. 2000;45:157-64.

18. Stewart 4th JH, Shen P, Levine EA. Intraperitoneal hyperthermic chemotherapy for peritoneal surface malignancy: current status and future directions. Ann Surg Oncol. 2005;12:765-77.

19. Pérez-Ruixo C, Valenzuela B, Peris JE, et al. Neutrophil dynamics in peritoneal carcinomatosis patients treated with cytoreductive surgery and hyperthermic intraperitoneal oxaliplatin. Clin Pharmacokinet. 2013;52:1111-25.

20. Davenport RA, Brohi K. Coagulopathy in trauma patients: importance of thrombocyte function? Curr Opin Anaesthesiol. 2009;22:261-6.

21. Jardim DL, Rodrigues CA, Novis YA, Rocha VG, Hoff PM. Oxaliplatin-related thrombocytopenia. Ann Oncol. 2012;23:1937-42.

22. Naesh O, Hindberg I, Friis J, et al. Platelet activation in major surgical stress: influence of combined epidural and general anaesthesia. Acta Anaesthesiol Scand. 1994;38:820-5.

23. Leibovitch I, Ben Chaim J, Raviv G, et al. Quantitative changes in platelet counts following major urological pelvic surgery. Eur Urol. 1993;24:350-4.

24. Naesh O, Friis JT, Hindberg I, Winther K. Platelet function in surgical stress. Thromb Haemost. 1985;54:849-52.

25. Becher RD, Shen P, Stewart JH, et al. Splenectomy ameliorates hematologic toxicity of hyperthermic intraperitoneal chemotherapy. J Gastrointest Oncol. 2011;2:70-6.

26. Valenzuela B, Nalda-Molina R, Bretcha-Boix P, et al. Pharmacokinetic and pharmacodynamic analysis of hyperthermic intraperitoneal oxaliplatin-induced neutropenia in subjects with peritoneal carcinomatosis. AAPS J. 2011;13:72-82.

27. Pérez-Ruixo C, Peris JE, Escudero-Ortiz V, et al. Rate and extent of oxaliplatin absorption after hyperthermic intraperitoneal administration in peritoneal carcinomatosis patients. Cancer Chemother Pharmacol. 2014;73:1009-20.

28. Beal SL, Sheiner LB, Boeckman AJ, (editors) NONMEM users guides. Ellicott City: ICON Development Solutions; 1989-2006.

29. Harker LA, Roskos LK, Marzec UM, et al. Effects of megakaryocyte growth and development factor on platelet production, platelet life span, and platelet function in healthy human volunteers. Blood. 2000;95:2514-22.

30. Krzyzanski W, Perez-Ruixo JJ, Harrold J. Pharmacodynamic model for chemoradiotherapy-induced thrombocytopenia in mice. J Pharmacokinet Pharmacodyn. 2015;42(6):709-20.

31. Roskos LK, Lum P, Lockbaum P, Schwab G, et al. Pharmacokinetic/pharmacodynamic modeling of pegfilgrastim in healthy subjects. J Clin Pharmacol. 2006;46:747-57.

32. Pérez-Ruixo JJ, Krzyzanski W, Hing J. Pharmacodynamic analysis of recombinant human erythropoietin effect on reticulocyte production rate and age distribution in healthy subjects. Clin Pharmacokinet. 2008;47:399-415.

33. Kaushansky K, Roth GJ. Platelets and megakaryocytes. In: Greer JP, Forester J, Luken J, et al. editors. Wintrobe's clinical hematology. 11th ed. Philadelphia: Lippincott Williams \& Wilkins; 2004. p. 605-50.

34. Krzyzanski W. Interpretation of transit compartments pharmacodynamic models as lifespan based indirect response models. J Pharmacokinet Pharmacodyn. 2011;38:179-204.

35. Folman CC, Ooms M, Kuenen BB, et al. The role of thrombopoietin in post-operative thrombocytosis. $\mathrm{Br} \mathrm{J}$ Haematol. 2001;114:126-33.
36. Kaser A, Brandacher G, Steurer W, et al. Interleukin-6 stimulates thrombopoiesis through thrombopoietin: role in inflammatory thrombocytosis. Blood. 2001;98:2720-5.

37. Zhang L, Beal SL, Sheiner LB. Simultaneous vs. sequential analysis for population PK/PD data I: best-case performance. J Pharmacokinet Pharmacodyn. 2003;30:387-404.

38. Savic RM, Karlsson MO. Importance of shrinkage in empirical Bayes estimates for diagnostics: problems and solutions. AAPS J. 2009;11:558-69.

39. Efron B, Tibshirani R. An introduction to the bootstrap. London: Chapman and Hall /CRC Press; 1993.

40. Brendel K, Comets E, Laffont C, et al. Metrics for external model evaluation with an application to the population pharmacokinetics of gliclazide. Pharm Res. 2006;23:2036-49.

41. Nguyen TH, Comets E, Mentré F. Extension of NPDE for evaluation of nonlinear mixed effect models in presence of data below the quantification limit with applications to HIV dynamic model. J Pharmacokinet Pharmacodyn. 2012;39:499-518.

42. Bergstrand M, Hooker AC, Wallin JE, et al. Prediction-corrected visual predictive checks for diagnosing nonlinear mixed-effects models. AAPS J. 2011;13:143-51.

43. Karlsson MO, Savic RM. Diagnosing model diagnostics. Clin Pharmacol Ther. 2007;82:17-20.

44. Desborough JP. The stress response to trauma and surgery. Br J Anaesth. 2000;85:109-17.

45. Hooker AC, Staatz CE, Karlsson MO. Conditional weighted residuals (CWRES): a model diagnostic for the FOCE Method. Pharm Res. 2007;24:2187-97.

46. Canda AE, Sokmen S, Terzi C, et al. Complications and toxicities after cytoreductive surgery and hyperthermic intraperitoneal chemotherapy. Ann Surg Oncol. 2013;20:1082-7.

47. Kaushansky K. Determinants of platelet number and regulation of thrombopoiesis. Hematology Am Soc Hematol Educ Program. 2009;147-152.

48. Warkentin TE. Thrombocytopenia caused by platelet destruction, hypersplenism, or hemodilution. In: Hoffman R, Benz EJ, Shattil SJ, et al. editors. Hematology: basic principles and practice. 6th ed. Philadelphia: Churchill Livingstone; 2013. p. 1895-912.

49. Schmitt A, Gladieff L, Laffont CM, et al. Factors for hematopoietic toxicity of carboplatin: refining the targeting of carboplatin systemic exposure. J Clin Oncol. 2010;28:4568-74.

50. Bruno E, Cooper RJ, Briddell RA, et al. Further examination of the effects of recombinant cytokines on the proliferation of human megakaryocyte progenitor cells. Blood. 1991;77:2339-46.

51. Drayer AL, Sibinga CT, Blom NR, et al. The in vitro effects of cytokines on expansion and migration of megakaryocyte progenitors. Br J Haematol. 2000;109:776-84.

52. Bender BC, Schaedeli-Stark F, Koch R, et al. A population pharmacokinetic/pharmacodynamic model of thrombocytopenia characterizing the effect of trastuzumab emtansine (T-DM1) on platelet counts in patients with HER2-positive metastatic breast cancer. Cancer Chemother Pharmacol. 2012;70:591-601.

53. Chalret du Rieu Q, Fouliard S, Jacquet-Bescond A, et al. Application of hematological toxicity modeling in clinical development of abexinostat (S-78454, PCI-24781), a new histone deacetylase inhibitor. Pharm Res. 2013;30:2640-53.

54. Bidus MA, Krivak TC, Howard R, et al. Hematologic changes after splenectomy for cytoreduction: implications for predicting infection and effects on chemotherapy. Int J Gynecol Cancer. 2006; 16:1957-62.

55. Oida T, Kano H, Mimatsu K, et al. Thrombocytosis following splenectomy: with or without additional organ resection. Hepatogastroenterology. 2012;59:1033-5.

56. Wang YM, Krzyzanski W, Doshi S, et al. Pharmacodynamicsmediated drug disposition (PDMDD) and precursor pool lifespan model for single dose of romiplostim in healthy subjects. AAPS J. 2010;12:729-40. 